\title{
Yes, we can use it: a formal test on the accuracy of low-pass nanopore long-read sequencing for mitophylogenomics and barcoding research using the Caribbean spiny lobster Panulirus argus
}

\author{
J. Antonio Baeza ${ }^{1,2,3}$ (D)
}

\begin{abstract}
Background: Whole mitogenomes or short fragments (i.e., 300-700 bp of the cox 1 gene) are the markers of choice for revealing within- and among-species genealogies. Protocols for sequencing and assembling mitogenomes include 'primer walking' or 'long PCR' followed by Sanger sequencing or Illumina short-read low-coverage whole genome (LC-WGS) sequencing with or without prior enrichment of mitochondrial DNA. The aforementioned strategies assemble complete and accurate mitochondrial genomes but are time consuming and/or expensive. In this study, I first tested whether mitogenomes can be sequenced from long-read nanopore sequencing data exclusively. Second, I explored the accuracy of the long-read assembled genomes by comparing them to a 'gold' standard reference mitogenome retrieved from the same individual using Illumina sequencing. Third and lastly, I tested if the long-read assemblies are useful for mitophylogenomics and barcoding research. To accomplish these goals, I used the Caribbean spiny lobster Panulirus argus, an ecologically relevant species in shallow water coral reefs and target of the most lucrative fishery in the greater Caribbean region.
\end{abstract}

Results: LC-WGS using a MinION ONT device and various de-novo and reference-based assembly pipelines retrieved a complete and highly accurate mitogenome for the Caribbean spiny lobster Panulirus argus. Discordance between each of the long-read assemblies and the reference mitogenome was mostly due to indels at the flanks of homopolymer regions. Although not 'perfect', phylogenetic analyses using entire mitogenomes or a fragment of the cox 1 gene demonstrated that mitogenomes assembled using long reads reliably identify the sequenced specimen as belonging to $P$. argus and distinguish it from other related species in the same genus, family, and superorder.

(Continued on next page)

Correspondence: jbaezam@clemson.edu

'Department of Biological Sciences, Clemson University, 132 Long Hall, Clemson, SC 29634, USA

${ }^{2}$ Smithsonian Marine Station at Fort Pierce, 701 Seaway Drive, Fort Pierce, Florida 34949, USA

Full list of author information is available at the end of the article

(c) The Author(s). 2020 Open Access This article is licensed under a Creative Commons Attribution 4.0 International License, which permits use, sharing, adaptation, distribution and reproduction in any medium or format, as long as you give appropriate credit to the original author(s) and the source, provide a link to the Creative Commons licence, and indicate if changes were made. The images or other third party material in this article are included in the article's Creative Commons licence, unless indicated otherwise in a credit line to the material. If material is not included in the article's Creative Commons licence and your intended use is not permitted by statutory regulation or exceeds the permitted use, you will need to obtain permission directly from the copyright holder. To view a copy of this licence, visit http://creativecommons.org/licenses/by/4.0/ The Creative Commons Public Domain Dedication waiver (http://creativecommons.org/publicdomain/zero/1.0/) applies to the data made available in this article, unless otherwise stated in a credit line to the data. 


\begin{abstract}
(Continued from previous page)
Conclusions: This study serves as a proof-of-concept for the future implementation of in-situ surveillance protocols using the MinION to detect mislabeling in P. argus across its supply chain. Mislabeling detection will improve fishery management in this overexploited lobster. This study will additionally aid in decreasing costs for exploring metapopulation connectivity in the Caribbean spiny lobster and will aid with the transfer of genomics technology to lowincome countries.
\end{abstract}

Keywords: Long-read sequencing, Nanopore, Lobster, Crayfish

\section{Background}

The mitochondrion is the energy-transducing organelle (a.k.a. the powerhouse) of eukaryotic cells. Other than playing an essential role in cellular energy provision, recent studies suggest that mitochondria are involved in other key cellular processes, including control of the cell cycle and cell growth [1,2]. The mitochondrion has its own genome, the mitochondrial DNA (mtDNA), most often comprised of a closed circular double-stranded DNA molecule $~ 15-20 \mathrm{kbp}$ in length. In animals (Metazoa), the structure and organization of the mtDNA is compact and well conserved within major clades, coding for a reduced set of intron-less protein coding genes (PCGs, $n=13$ ) that belong to different enzyme complexes of the oxidative phosphorylation system, 22 transfer RNAs (tRNAs), and the two subunits (12S [rrnS] and $16 \mathrm{~S}[\mathrm{rrnL}])$ of the mitochondrial ribosomal RNA $[1,3]$. Certainly, exceptions to the aforementioned organization exist; mtDNA comprised of one or more linear molecules only or along with circular molecules have been reported in some invertebrate clades (e.g., Anthozoa: Meduzoa, Insecta: Phthiraptera) while in others, limited or moderate single- or multi-gene block deletions, duplications, inversions, and/or translocations are known [3]. Furthermore, a recent study has reported a parasite that has secondary lost the mitochondrial genome in its entirety (i.e., the dinoflagellate Amoebophrya ceratii - [4]). The mitochondrial genes are either lost or encoded in the nucleus in $A$. ceratii [4].

When present, multiple copies of mitochondria exist within each metazoan cell. mtDNA inheritance is maternal-only (clonal), and thus the mitochondrial chromosome behaves as a single non-recombining locus (but see [5] for a review of doubly uniparental inheritance and [6] for mtDNA paternal leakage). The mutation rate of mtDNA is high compared to most nuclear markers and has been assumed to evolve in a nearly neutral fashion ([3, 7], but see [8]). Given these feats, the entire or a reduced representation (i.e., one or a few PCG fragments) of the mtDNA is straightforward to sequence and became the marker of choice for revealing within- and among-species genealogical relations during past decades [9]. Furthermore, with the advent of second- (i.e., Illumina short-reads) and third-generation (long-read) sequencing technologies, whole mitochondrial genomes have been used for phylogeographic and phylogenomic analyses ([10-12] and references therein) instead of only a few fragments (i.e., cox1, cob, 12S, 16S). An ever increasing number of studies reporting the structural and functional organization of animal mitochondrial genomes is available in NCBI's Genbank (https://www.ncbi.nlm.nih.gov/genbank/) permitting the integration of mtDNA topological features (i.e., deletions, insertions, translocations, and overall gene synteny) concomitantly with sequence similarity to inform phylogenetic relationships among species at multiple taxonomic levels (e.g., [11, 13-15]).

Herein, I focus on testing a strategy for the rapid sequencing and assembling of mitochondrial genomes (mtDNA) profiting from third generation sequencing technologies. For more than 20 years, the standard protocol for sequencing and assembling mitochondrial genomes was based either on 'primer walking' or 'long PCR' and cloning plus Sanger sequencing [16]. During the last decade, however, second generation sequencing technologies have been used for low-coverage (= lowpass) whole genome sequencing (i.e., genome skimming) with or without prior mitochondrial enrichment to assemble mitochondrial chromosomes (e.g., [13]). This strategy often results in the assembly of complete and totally accurate mitochondrial genomes but it is time consuming, with projects often lasting from weeks to months from initial DNA purification to genome assembly and annotation [11, 13-15]. Rapid and simple library preparation, sequencing, and assembly of any DNA marker, including complete mitochondrial genomes, are desirable to solve a plethora of problems in conservation biology, including resource management. For instance, rapid DNA recovery is of utmost importance for researchers focusing on real-time genomic surveillance of pathogens [17] or the in-situ identification and detection of mislabeling in the supply chain of biological commodities [18]. Mitochondrial genome sequencing based on short reads is not the optimal solution for these studies or other studies requiring the speedy recovery of molecular markers.

An alternative to short-read data for mitochondrial genome sequencing is the use of third generation 
sequencing technology; long reads produced by devices such as those manufactured by Pacific Biosciences (PacBio) and Oxford Nanopore Technologies (ONT). PacBio and ONT devices are currently capable of sequencing long molecules with an average of $\sim 10-20 \mathrm{kbp}$ and up to 1-2 Mbp [19]. The main problem with third generation sequencing technologies is the high initial sequence error rate; much greater than that of Illumina sequencing (PacBio $=11-15 \%$ and $\mathrm{ONT}=5-15 \%$ versus $0.3 \%$ initial sequencing error rate reported for Illumina reads $[20,21])$. Furthermore, a second major problem with PacBio sequencing is that library preparation and sequencing are considerably more expensive and time consuming compared to Illumina sequencing [20]. In contrast to PacBio, nanopore library preparation and sequencing is relatively quick and straightforward, and the sequencing device itself is inexpensive compared to that of PacBio and Illumina machines [19]. Indeed, nanopore sequencing can be considered a disruptive technology with the potential of breaking cost-barriers to provide relatively cheap sequencing for researchers in moderateand low-income countries that are in need of rapid retrieval of molecular markers for answering a wide variety of biological conservation problems. The high initial error rate of nanopore long reads is currently corrected using complex in-silico sequence 'polishing' algorithms ([19] and references therein). Considering that mitochondrial genomes are short, circular, non-repetitive, haploid chromosomes with low GC content, the assembly of these genomes should be straightforward using third generation sequencing devices.

Most recently, long- and short-read datasets have been used collectively for the so-called 'hybrid assembly' of a variety of prokaryotic organisms ([22] and references therein) as well as for assembly of mitochondrial [23$25]$, chloroplast [23, 26, 27], and nuclear genomes in various eukaryotes (e.g., plants: [28]; animals: [29] and references therein). The assembly of genomes using long reads alone is rare but is becoming widespread; long reads have been used for de novo or reference-based assembly of viral [22], bacterial [22, 30], and relatively small and large eukaryotic genomes (e.g., de novo genome assembly of the eel Anguilla anguilla [31] and Homo sapiens [19], respectively) in recent years. In the case of animal mitochondrial genomes, hybrid assemblies have been successful in clawed lobsters (Homarus gamarus - [24]) and land crabs (Gecarcoidea natalis [25]). To the best of the author's knowledge, only a single study that employed a de novo assembly strategy using long reads alone produced a complete and fully accurate mitochondrial genome in a neotropical rodent (Melanomys caliginosus - [32]). Importantly, the latter study benchmarked the long-read mitochondrial genome assembly using only two relatively short protein coding gene fragments obtained via Sanger sequencing [32]. Only after considerable manual curation, the authors (see [32]) claimed the assembly of a complete and fully accurate genome. However, the algorithm used for the final manual assembly curation was not explained in detail. Benchmarking of long-read assemblies with full reference genomes produced with short-read Illumina or Sanger sequencing is of utmost importance: it will aid in optimizing protocols focusing on the rapid de novo assembly of mitochondrial genomes using third generation sequencing technologies alone.

The aims of this study were threefold. First, I tested whether a mitochondrial genome can be sequenced and assembled from long-read nanopore sequencing data alone using both a de novo and a reference-based strategy. Second, I explored the quality (i.e., accuracy) of the long-read assembled genomes by comparing them to a 'gold' standard mitochondrial genome retrieved from the same individual but generated using short-read Illumina sequencing data. Sequence accuracy was explored for different long-read assembly pipelines with multiple metrics including completeness, identity, and coverage. Furthermore, a detailed quantitative analysis of error type in long-read assemblies was conducted. Third and lastly, I tested if the de novo and reference-based longread assemblies are useful for mitophylogenomics and barcoding research. I specifically assessed whether longread assemblies contain phylogenomic information that permit to reliably identify the sequenced specimen as belonging to $P$. argus and distinguish it from other closely and distantly related species in the same genus, family, and superorder.

To accomplish these goals, I used the Caribbean spiny lobster Panulirus argus, an ecologically relevant species in shallow water coral reefs [33] and target of the most lucrative fishery ( 1B USD) in the greater Caribbean region [34] (Fig. 1). Panulirus argus is fully exploited or overexploited across its entire geographic range [34] and mislabeling of this marine resource across multiple steps in its supply chain is common (JA Baeza, pers. obs.). Despite its ecological importance, commercial value, and mislabeling in the trade of $P$. argus, only a few (but increasing) number of genomic resources exist for this species [13, 35-38]. The development of genomic resources are of utmost importance as they will improve the understanding about the biology of $P$. argus while also aiding in fishery management and conservation strategies using relative cheap molecular markers.

\section{Results}

Mitochondrial genome assembly of Panulirus argus using short reads

The mitochondrial chromosome of $P$. argus was assembled and circularized in NOVOPlastly with an average 

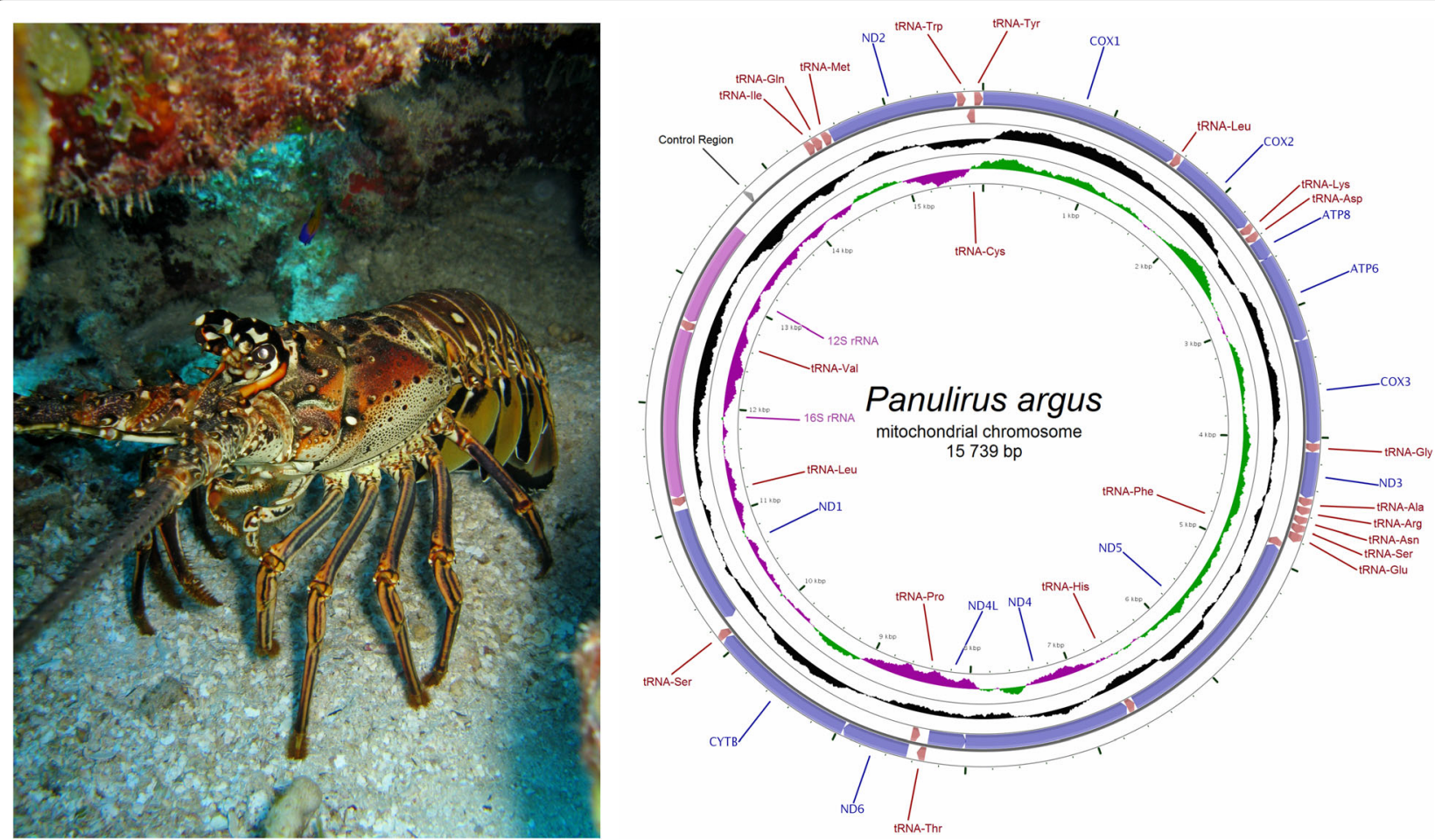

Fig. 1 The Caribbean spiny lobster Panulirus argus (left) and circular genome map of Panulirus argus mitochondrial DNA (right). The map is annotated and depicts 13 protein-coding genes (PCGs), 2 ribosomal RNA genes (rrnS [12S ribosomal RNA] and rrnL [16S ribosomal RNA]), 22 transfer RNA (tRNA) genes, and the putative control region. The inner circle depicts GC content along the genome

coverage of 710x. The complete mitochondrial genome of $P$. argus (identical to GeneBank accession number MH068821) was $15,739 \mathrm{bp}$ in length. Annotation in MITOS and MITOS2 indicated that the mtDNA of $P$. argus was comprised of 13 protein-coding genes (PCGs), 2 ribosomal RNA genes (rrnS [12S ribosomal RNA] and rrnL [16S ribosomal RNA]), and 22 transfer RNA (tRNA) genes. Most of the PCGs and tRNA genes were encoded on the L-strand. Only 4 PCGs (nad5, nad4, nad4l, and nad1) and 8 tRNA genes $(\operatorname{trnF}, \operatorname{trnH}, \operatorname{trn} P, \operatorname{trnL} 1, \operatorname{trn} \mathrm{V}, \operatorname{trn} \mathrm{Q}, \operatorname{trnC}$, trnY) were encoded in the $\mathrm{H}$-strand. The 2 ribosomal RNA genes were encoded in the $\mathrm{H}$-strand (Fig. 1). A single relatively long inter-genic space involving $801 \mathrm{bp}$ in the mitochondrial genome of $P$. argus was assumed to be the D-loop/Control Region. The gene order observed in $P$. argus is identical to that reported before in the genus Panulirus and corresponds to the presumed Pancrustacean (Hexapoda + Crustacea) ground pattern [13].

\section{Mitochondrial genome assembly of Panulirus argus using long reads}

The pipeline Canu, unexpectedly, did not assemble any circular molecule either with default setting or with parameters modified to optimize the retrieval of small circular sequences from data with uneven coverage. In contrast to Canu, all other pipelines (i.e., Unicycler, Flye, and Rebaler with and without 'extra' polishing with Medaka) assembled and circularized the mitochondrial genome of $P$. argus as indicated after examination of contigs in the software Bandage and contigs blasts against the NCBI nucleotide non-redundant database (all circular contigs matched the mitochondrial genome of $P$. argus available in GenBank with e-values $<<1 \mathrm{e}^{-10}$ ). Blasting of linear contigs generated by Unicycle and Flye did not match any other mitochondrial sequences belonging to the genus Panulirus available in GenBank.

All long-read assemblies, either de novo (i.e., Unicycler and Flye) or reference-based (i.e., Rebaler) with or without extra polishing with Medaka, varied in length between 15, $661 \mathrm{bp}$ (Flye with 10 polishing cycles and no extra polishing with Medaka) and 15,725 bp (Rebaler using $P$. versicolor as a reference and with extra polishing with Medaka). Nonetheless, all long-read assembled mitochondrial genomes were shorter (range: 14-77 bp) than the reference genome assembled with short reads in NOVOPlasty. Furthermore, all long-read assembled mitochondrial genomes that were not extra-polished with the software Medaka were shorter than those treated with the latter tool (range non-polished: $15,661-15,720 \mathrm{bp}$; range polished: $15,717-15,725 \mathrm{bp}$ ).

All long-read assemblies were identical (e.g., Flye with 1 polish round = with 5 polish rounds; Unicycler-normal = -bold $=-$ conservative) or very similar to each other with 
Table 1 Accuracy metrics for different de novo and reference-based mitochondrial genome assemblies using nanopore long reads exclusively in the Caribbean spiny lobster Panulirus argus

\begin{tabular}{|c|c|c|c|c|c|}
\hline Assembly Pipeline & Contigs & Length & Coverage & p-dist & Errors \\
\hline Canu - general & - & - & - & - & - \\
\hline Canu - specific & - & - & - & - & - \\
\hline Flye $+1 p$ & circular & 15,662 & $35 x$ & 0.000191632 & 77 \\
\hline Flye $+1 p+$ Medaka & circular & 15,717 & $35 x$ & $6.37024 \mathrm{E}-05$ & 51 \\
\hline Flye $+5 p$ & circular & 15,662 & $35 x$ & 0.000191632 & 77 \\
\hline Flye $+5 p+$ Medaka & circular & 15,717 & $35 x$ & $6.37024 \mathrm{E}-05$ & 51 \\
\hline Flye $+10 p$ & circular & 15,661 & $35 x$ & 0.000191632 & 76 \\
\hline Flye $+10 p+$ Medaka & circular & 15,717 & $35 x$ & $6.37024 \mathrm{E}-05$ & 51 \\
\hline Unicycler - N & circular & 15,718 & $0.411 x^{a}$ & 0.000637552 & 69 \\
\hline Unicycler - N + Medaka & circular & 15,724 & $0.411 x^{a}$ & 0.000637552 & 53 \\
\hline Unicycler - B & circular & 15,718 & $0.411 x^{a}$ & 0.000637552 & 59 \\
\hline Unicycler - B + Medaka & circular & 15,724 & $0.411 x^{a}$ & 0.00012738 & 53 \\
\hline Unicycler - C & circular & 15,718 & $0.411 x^{a}$ & 0.00012738 & 59 \\
\hline Unicycler - C+ Medaka & circular & 15,724 & $0.411 x^{a}$ & 0.00012738 & 53 \\
\hline Rebaler - P. versicolor & circular & 15,709 & $30.06 x$ & 0.000191253 & 70 \\
\hline Rebaler - P. versicolor + Medaka & circular & 15,725 & $30.06 x$ & 0.000191241 & 55 \\
\hline Rebaler - $P$. cygnus & circular & 15,720 & $34.13 x$ & 0.000318756 & 72 \\
\hline Rebaler - P. cygnus + Medaka & circular & 15,723 & $34.13 x$ & $6.36862 \mathrm{E}-05$ & 57 \\
\hline Rebaler - P. argus & circular & 15,713 & $40.75 x$ & $6.36821 \mathrm{E}-05$ & 69 \\
\hline Rebaler - P. argus + Medaka & circular & 15,721 & $40.75 x$ & 0.00012738 & 55 \\
\hline Reference mtDNA & circular & 15,739 & $720 x$ & - & - \\
\hline
\end{tabular}

anicycler normalises the depth of contigs to the median value

$p$-values ranging between $6.3613 \times 10^{-5}$ (Rebaler-Panulirus cygnus based versus Rebaler-Panulirus argus based) and $7.0306 \times 10^{-4}$ (Flye with 10 polishing rounds without extra polishing with Medaka versus Unicycler-normal, Unicycler-bold, and Unicycler-conservative without extra polishing with Medaka) when dissimilar. Identity was also very high as all assemblies were a close match to the reference genome with $p$-values ranging between $6.36821 \times$ $10^{-5}$ (reference compared to Rebaler using P. cygnus as a reference with extra polishing with Medaka) and $6.3755 \times$ $10^{-4}$ (reference compared to Unicycler-normal, -bold, and -conservative, all without extra polishing with Medaka) (Table 1).

Alignment of the different long-read assemblies to the reference genome revealed that discordance between each of the long-read assemblies and the reference assembly was mostly due to indels at the flanks of homopolymer regions comprising all four nucleotide types (Fig. 2). The number of single nucleotide homopolymer deletions was by far the most common error detected in all long-read assemblies followed by single nucleotide homopolymer insertions. Errors due to double homopolymer insertions and deletions, and single insertions were moderately abundant, in particular in the Unicycle and
Rebaler assemblies (Fig. 2). Errors due to triple homopolymer deletion, single deletion, short insertions $(\leq 5$ bp), and substitutions were less common. Triple, quadruple, and quintuple homopolymer insertions, and short deletions $(\leq 3 \mathrm{bp}$ ) were rare. In general, less homopolymer deletions were observed in Unicycler and Rebaler than in Flye assemblies and larger number of homopolymer inserts were observed in Rebaler and Unicycler than Flye assemblies.

The main effect of extra-polishing with Medaka, across de novo and reference-based mitochondrial genomes, was a decrease in the number of homopolymer deletions. This effect was particularly evident for mitochondrial genomes assembled with the pipeline Flye in which homopolymer deletions decreased by more than half when Medaka extra-polishing was applied. In general, extra-polishing with the program Medaka resulted in increased accuracy, especially for the assemblies using the software Flye.

Overall, accuracy of the assembled genomes using long reads was most similar when assessed in terms of completeness (contigs), length, coverage, identity, and sequence errors. Long-read genome accuracy was also very high, although not $100 \%$, as detected using the shortread assembled genome as a reference (Table 1). 


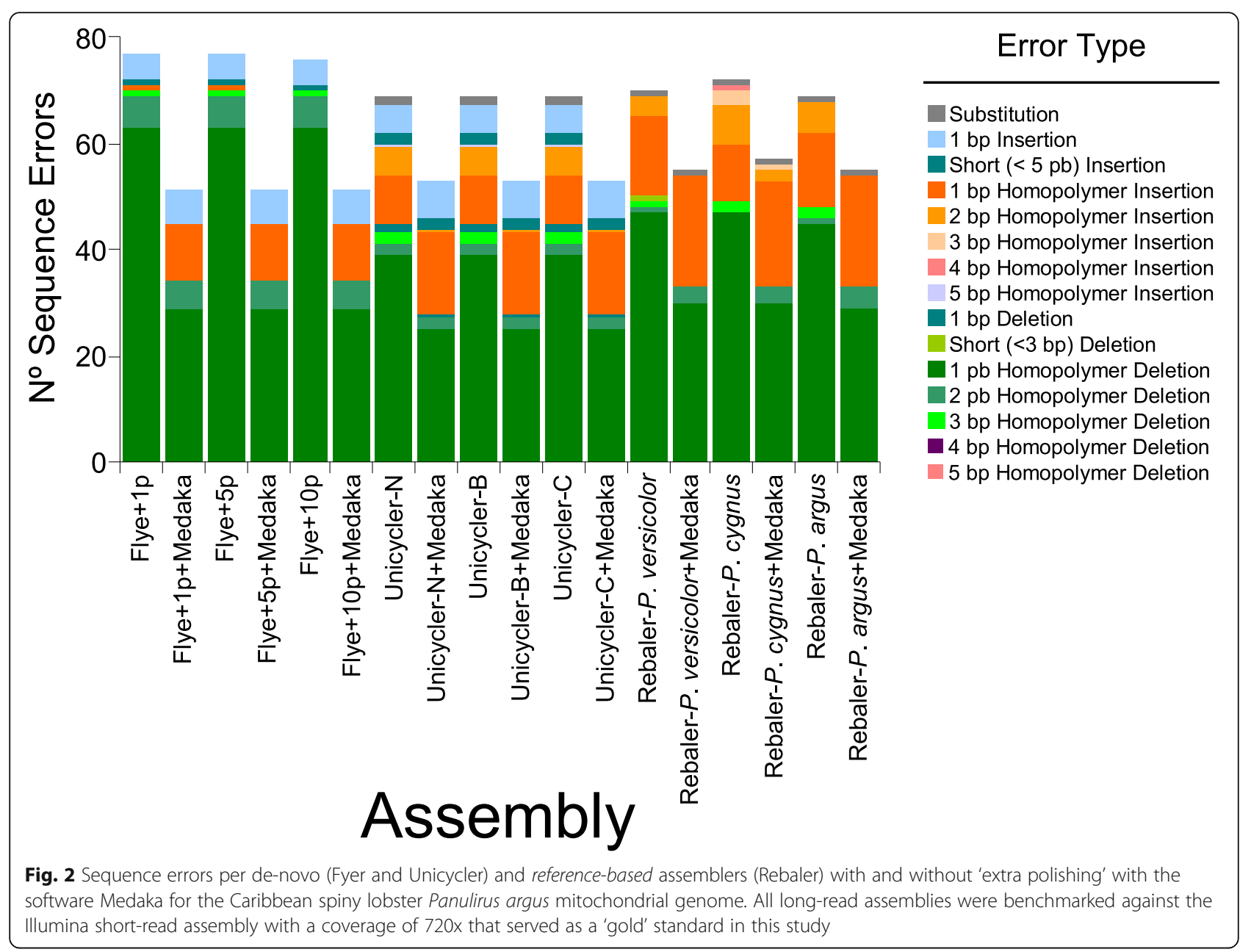

\section{Annotation of mitochondrial genome assemblies using long reads}

Annotation of long-read assembled mitochondrial genomes, either de novo or reference-based with or without extra-polishing with Medaka, indicated that gene number and synteny were identical to that of the reference genome (Fig. 3). Each long-read mitochondrial genome comprised 13 PCGs, $12 \mathrm{~S}$ and $16 \mathrm{~S}$ ribosomal RNA genes, and 22 tRNA genes. Importantly, all but 1-2 of the genes did have at least one internal stop codon (and usually more) that interrupted their open reading frames. Although highly accurate, the errors contained in each long-read assembled mitochondrial genome precluded generating a reliable annotation with MITOS and MITOS2 (Fig. 3).

\section{Mitophylogenomics using long-read mitochondrial genome assemblies}

In the ML molecular phylogenetic tree (42 terminals, 11 , 187 nucleotide characters, 6340 informative sites), the totality $(n=18)$ of the long-read assembled mitochondrial genomes and the short-read assembled reference genome clustered together into a single monophyletic clade strongly supported by the bootstrap support value from the ML analysis (bootstrap value [bv] = 100) (Fig. 4). The tree also placed P. argus (all long-read and reference short-read assemblies) in a monophyletic clade with $P$. japonicus and $P$. cygnus, in agreement with previous phylogenetic studies using a combination of partial mitochondrial and nuclear genes (Ptacek et al. 2000) (Fig. 4). Additional well supported clades within the Achelata included the genera Ibacus and Scyllarides. Unexpectedly, the tree did not confirm the monophyly of the Achelata given the position of Remiarctus bertholdii that clustered together (but only with moderate to low support) with representatives of the order Polychelidae instead of with the remainder representatives of the order Achelata. Support values did not decrease considerably towards the root of the phylogenetic tree and several nodes located near the root of the tree were well supported (Fig. 4). The above suggest that mitochondrial 


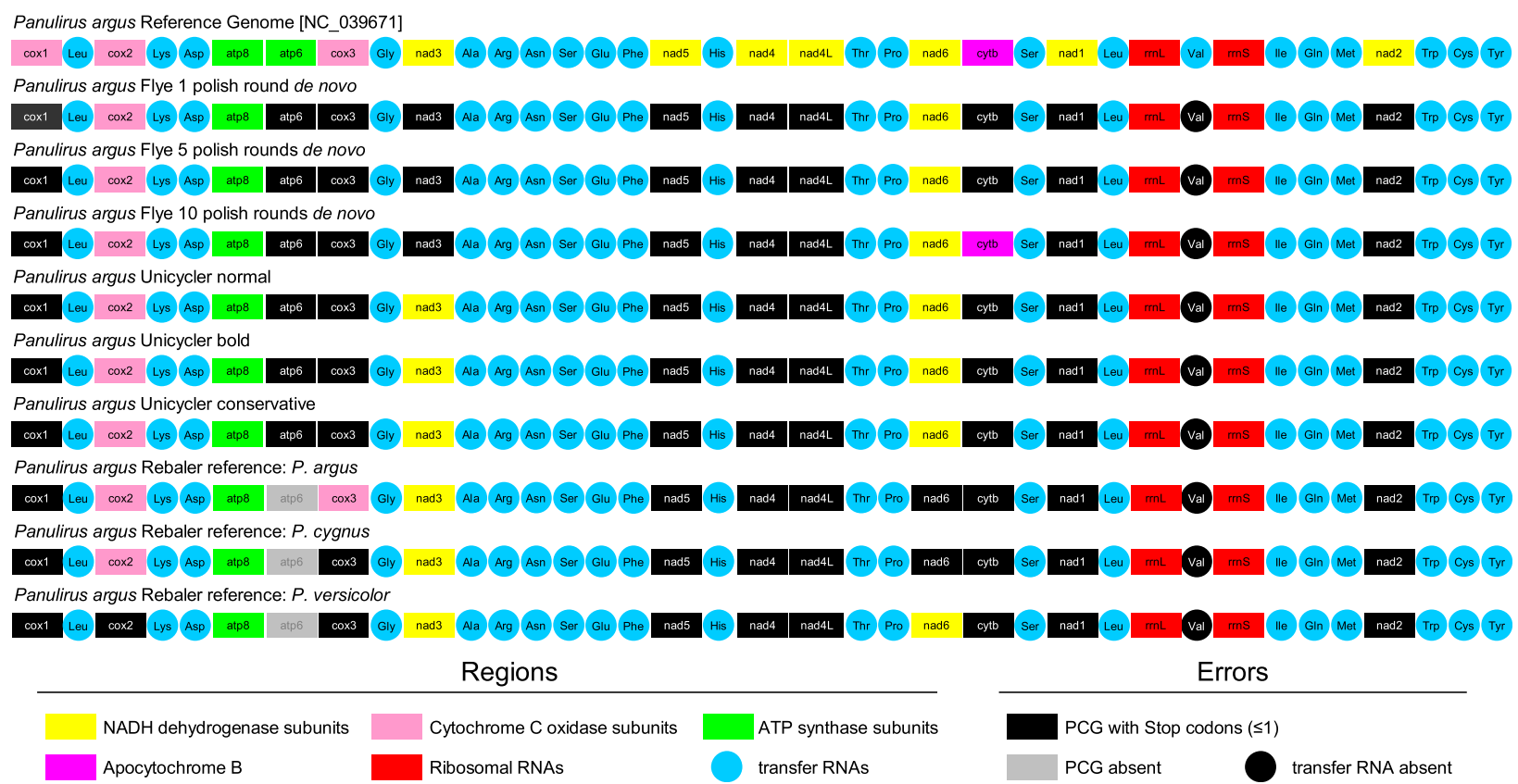

Fig. 3 Annotation of de novo (Fyer and Unicycler) and reference-based (Rebaler) assemblies with and without 'extra polishing' with the software Medaka for the Caribbean spiny lobster Panulirus argus mitochondrial genome

genomes alone will likely have enough phylogenetic information to reveal relationships at higher taxonomic levels within the Crustacea, including the Achelata.

\section{Barcoding using long-read mitochondrial genome assemblies}

In the different phylogenetic analyses based on the first, second, and third portion of the cox 1 gene, the aligned molecular data matrix comprised, respectively, 500, 500, and 539 characters, of which 278, 223, and 369 were parsimony informative, for a total of 1899, 185, and 210 terminals belonging to spiny lobsters (genus Panulirus), other related congeneric and confamiliar species, plus outgroup terminals from the superorder Achelata (Fig. 5). In all ML molecular phylogenetic trees (Fig. 5), the totality $(n=18)$ of the long-read assembled mitochondrial genomes and the short-read assembled reference genome clustered together into a single monophyletic clade strongly supported by the bootstrap support values from the ML analyses (bootstrap value [bv] $=100$ in all three cases). Importantly, in the ML analysis of the first dataset $(1-500 \mathrm{bp})$ that included the largest number of terminals among the three analyses, this robustly supported clade comprising long-read assembled mitochondrial genomes and the short-read reference assembly plus a total of 340 sequences belonging to $P$. argus retrieved from Genbank clustered together into another monophyletic clade that was strongly supported $[\mathrm{bv}=98]$ (Fig. 5). Other well supported clades included $P$. interruptus, the
P. penicillatus species complex, the $P$. elephas $+P$. mauritanicus species complex, and various other species belonging to the genus Panulirus in the superorder Achelata (Fig. 5). Note that lower bootstrap values were observed towards the root of the tree as is expected considering that short fragments of the cox 1 gene should not have any phylogenetic informativeness to resolve deep genealogical relationships.

In conclusion, although not completely accurate, longread mitochondrial genomes can reliably identify the sequenced specimen as belonging to $P$. argus and can differentiate the specimen from other closely and distantly related species in the same genus, family, and superorder.

\section{Discussion}

This study demonstrates that complete mitochondrial genomes can be assembled using nanopore sequencing data alone using both de novo and reference-based approaches. Using low-coverage whole genome shot-gun long-read sequencing, most of the pipelines used herein retrieved a complete mitochondrial genome, as shown when these long-read assemblies were compared to a high-coverage 'reference' assembly generated from the same individual but with Illumina short reads. Canu was the only pipeline that failed to assemble and circularize the studied mitochondrial chromosome even when parameters were customized to optimize the assembly of short circular molecules ([39], see also [40]). Earlier versions of Canu were known to 'have trouble' assembling 


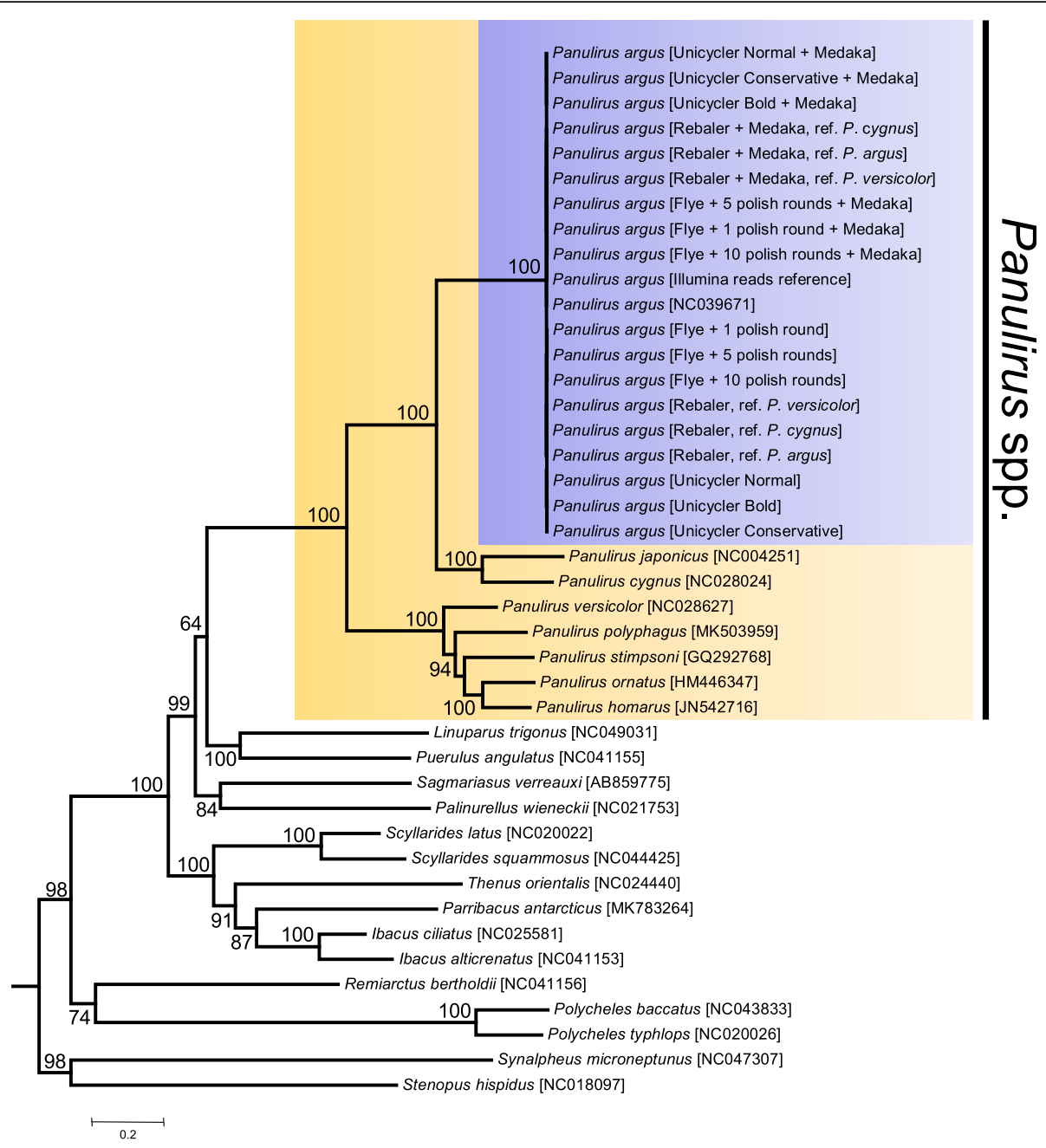

Fig. 4 Mitophylogenomic analysis of the order Achelata, including mitochondrial genomes of Caribbean spiny lobster Panulirus argus assembled with long reads alone and short reads ('gold standard')

short circular molecules i.e., plasmids, in datasets with uneven coverage [39]. However, the most recent version of Canu (used in this study) dynamically selects poorly represented sequences to avoid missing short circular sequences [39]. Still, the problem persists based on the results from this study. Previous studies using Canu have successfully assembled mitochondrial genomes [32] and larger circular chromosomes (i.e., chloroplasts - [40], bacteria - [22]). Importantly, in this study, Canu not only failed to assemble the mitochondrial genome of $P$. argus but also took 20-30 times longer to execute compared to the other pipelines that assembled and circularized the studied mitochondrial genome in $<2 \mathrm{~h}$. The current version of Canu automatically detect available resources in a computer or cluster and configures itself to run 'efficiently' using those resources [39]. Thus, Canu has the potential to negatively impact pipelines activated by other users using the same cluster at the same time. The results from this study suggest that long-read assemblers like Unicycler, Flye, and Rebaler should be preferred over Canu when the goal is to assemble mitochondrial genomes.

For those pipelines that were successful in assembling the mitochondrial genome of $P$. argus, the quality (i.e., accuracy) of the assembly was high. All pipelines other than Canu circularized the genome with a coverage > 20-30x and sequence (mtDNA) identity, as measured by p-distance, was very high. Importantly, a detailed quantitative comparison of error types in long-read assemblies indicated that the most usual errors were deletions in homopolymer runs, in line with that observed by [39] when assembling chloroplast genomes. The commonality of this type of error also fits with expectations for non-random Nanopore sequencing errors [41]. Note that indel errors in homopolymer runs is not an issue exclusive to long-read sequencing; it occurs in short-read platforms too, but at a much smaller rate [41]. Errors due to double homopolymer insertions and deletions, 


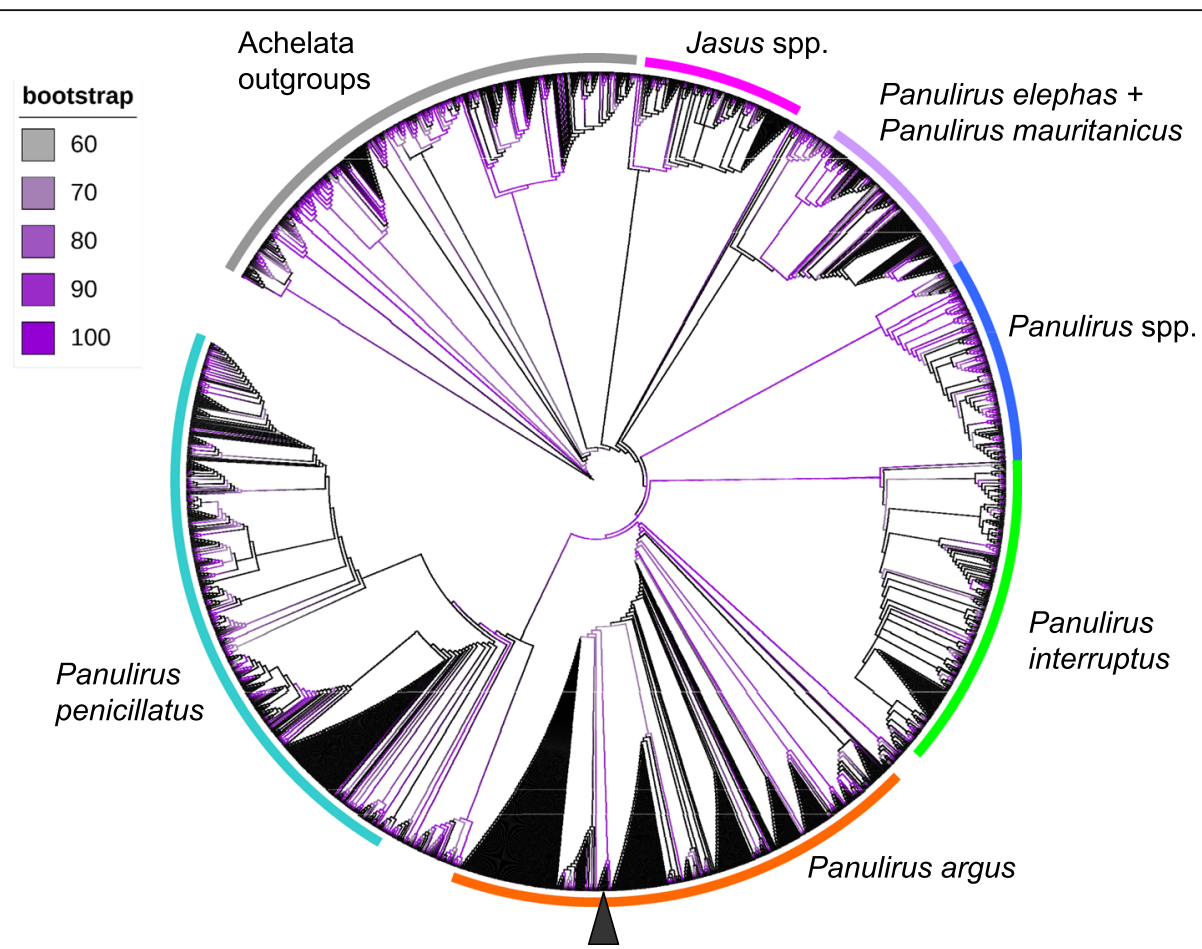

Fig. 5 Barcoding analysis of the order Achelata using a 500 bp fragment of the cox 1 gene, including cox 1 gene fragments retrieved from mitochondrial genomes of the Caribbean spiny lobster Panulirus argus assembled with long reads alone and short reads ('gold standard') plus 1884 other terminals belonging to the order Achelata retrieved from Genbank

and single insertions were moderately abundant, in particular in the Unicycle and Rebaler assemblies while errors due to triple homopolymer deletion, single deletion, short insertions $(\leq 5 \mathrm{bp})$, and substitutions were less common. Triple, quadruple, and quintuple homopolymer insertions, and short deletions $(\leq 3 \mathrm{bp}$ ) were rare. Unfortunately, the only previous study assembling a mitochondrial genome with nanopore long reads alone did not characterize errors [32]. For the MinION, independent error-assessments are rather scant $[41,42]$. The present benchmarking results are expected to inform software usage as well as the future development of nanopore long-read sequencing technology.

No major differences in accuracy were observed among the different pipelines, either de novo or reference-based, that succeeded in assembling the mitogenome of $P$. argus into a complete circular molecule. Also, in the case of reference-based assembly strategies, no major effect of the choice of a reference (closely or distantly related congeneric species) was observed. Only a slight but detectable decrease in accuracy occurred when the reference genome was from the distantly related species $P$. versicolor. To compare, a recent study that assembled chloroplasts using nanopore long reads did report a decrease in accuracy when the genome used as a reference belonged to a distantly related species [40]. In this study, obvious differences among assemblies were observed only when extra polishing with the pipeline Medaka was applied, in line with expectations; the pipeline Medaka should result in improved accuracy given the state-of-the-art complex neural network applied by this tool while searching for sequence consensus (https://github.com/nanoporetech/medaka). Long-read nanopore technology as well as PacBio have been steadily decreasing their initial error rate during the last 5 years [21]. Further improvements with the long-read technology are expected to result in complete and totally accurate mitochondrial and other larger genomes in the next years.

\section{Utility of long-read genomes for mitophylogenomics and barcoding research}

Long-read nanopore sequencing concomitantly with the state-of-the-art bioinformatics pipelines used in this study resulted in the assembly of a complete and highly accurate mitochondrial genome in the Caribbean spiny lobster $P$. argus. Importantly though, the few observed errors were enough to disrupt the ORF in almost every PCG when the mitogenomes were annotated using state-of-the-art pipelines [43]. Although the structural annotation (gene synteny) of the long-read mitochondrial genomes were identical to that of the reference genome, at least one and often multiple stop codons were found in the ORF of most PCGs, resulting in a highly inaccurate functional 
annotation. The latter result forces the fair conclusion that the current technological state of nanopore sequencing with the MinION ONT does not permit the assembly of mitochondrial genomes with an accuracy that can be used to inform studies on mtDNA evolution and/or applied medical research (i.e., mitochondrial disease dynamics in humans). The assembly of accurate mitochondrial genomes is of utter importance for conducting reliable a posteriori analyses, including but not limited to codon and nucleotide usage, selective pressures in PCGs, and secondary structure of tRNAs, among others, that inform mtDNA function and evolution.

Although 'imperfect' (= not totally accurate), I argue that the assembled genomes could be reliable enough for the identification of the sequenced specimen as $P$. argus and to differentiate the specimen from other representatives belonging to the same genus and family within the Decapoda Achelata (clawed lobsters). If the assembled mitochondrial genome or fragments of it (i.e., cox1 gene) are useful for phylogenomics and barcoding studies, then I predicted that the entire mtDNA as well as a fragment of it (i.e., the $\operatorname{cox} 1$ gene alone) produced by the de novo and reference-based assemblies will cluster with the short-read reference assembly as well as with other $\operatorname{cox} 1$ fragments from the same species available in GenBank and will segregate from other closely related congeneric and confamiliar species (and even from more distantly related species) in the same order Achelata. Note that most $\operatorname{cox} 1$ gene fragments and complete mitochondrial genomes available in GenBank are retrieved via Sanger sequencing or assembled with Illumina short-reads, respectively (https://www.ncbi.nlm. nih.gov/genbank/).

Supporting the notion that long-read retrieved genomes can be used in mitophylogenomics and barcoding studies, in a first phylogenetic analysis using PCGs, the totality of the long-read assembled mitochondrial genomes and the short-read assembled reference genome clustered together into a single monophyletic clade. The phylogenetic tree also placed $P$. argus (all long-read and reference short-read assemblies) in a monophyletic clade with $P$. japonicus and $P$. cygnus, in agreement with previous phylogenetic studies using a combination of partial mitochondrial and nuclear genes [44] or complete mitochondrial genomes [11]. Furthermore, and perhaps more importantly, in a second set of analyses using only a 500 bp fragment of the cox 1 gene, the long-read genomes together with the short-read reference genome clustered into a single well supported clade comprising more than 500 other sequences from the same species. This latter clade segregated from others containing more than 1000 sequences from other closely related (congeneric and confamiliar species) and other more distantly related species belonging to the order Achelata. These results support the notion that mitochondrial genomes (including those assembled using long reads) alone have enough phylogenetic information to reveal relationships at higher taxonomic levels within the Crustacea order Decapoda (see [11-13]). Additionally, these results are encouraging as they suggest that nanopore sequencing technology can also be used to answer major problems related to the conservation and management of the Caribbean spiny lobster.

In conclusion, although not completely accurate, longread mitochondrial genomes can reliably identify the sequenced specimen as belonging to $P$. argus and can differentiate the specimen from other closely and distantly related species in the same genus, family, and superorder. This study serves as a proof-of-concept demonstrating that the design of an in situ surveillance protocol for detecting mislabeling of $P$. argus at multiple steps of its supply chain is feasible with the MinION ONT. Such surveillance and identification of mislabeling (involuntary or not) will improve fishery management and inform conservation strategies of this overexploited resource.

Lastly, these results also suggest that retrieval of mitochondrial genomes using nanopore sequencing will result in decreased sequencing costs when examining connectivity among populations of $P$. argus across the greater Caribbean region. Understanding the connectivity patterns in this species will help reveal source-andsink metapopulations dynamics, migration patterns, and ultimately, benefit the design and implementation of marine protected areas [45]. Previous studies have based connectivity inferences using either a limited set of mitochondrial markers (cox1 gene fragment - [46]) or microsatellites [45]. Together with a recently developed panel of nuclear SNPs [36], entire mitochondrial genomes will permit revealing fine-grain spatial and temporal patterns of connectivity in $P$. argus across its range of distribution (from North Carolina, USA to Brazil). Ideally, such studies need to be implemented in situ together with local biologists across the greater Caribbean region and nanopore sequencing might be able to deliver rapid and cheap genetic marker retrieval to researchers in lowand moderate-income countries.

\section{Conclusion}

In conclusion, using nanopore long-read sequencing and various bioinformatics pipelines, this study assembled a complete and highly accurate mitochondrial genome for the Caribbean spiny lobster $P$. argus, a keystone species in shallow water coral reefs and target of the most lucrative fishery in the greater Caribbean region. The assembled genomes were 'imperfect' but permitted to identify reliably the sequenced specimen as belonging to $P$. argus and differentiate the specimen from other closely and distantly related species in the same genus, family, and 
superorder. This new genomic resource will contribute to the better understanding of meta-population connectivity in this overexploited species and will guide future strategies for sequencing the whole genome of $P$. argus. Lastly, this study will facilitate the transferring of genomic technologies to low-income countries in the greater Caribbean, allowing the mislabeling of overexploited lobsters to be monitored.

\section{Methods}

\section{Sampling of Panulirus argus}

Field collection was approved by FWCC (permit number: SAL-11-1319-SR). One adult female of $P$. argus was collected in July 2017 by hand while SCUBA diving from a patch reef on the ocean side of Long Key (N24.49' 26"; W80 $48^{\prime} 48^{\prime \prime}$ ), Florida, USA and transported alive to Clemson University, Clemson, SC. In the laboratory, the specimen was maintained in a $500 \mathrm{~L}$ circular polyethylene container. The specimen was first placed in a refrigerator $\left(-5^{\circ} \mathrm{C}\right)$ to render it unconscious and then euthanized and maintained in a freezer $\left(-20^{\circ} \mathrm{C}\right)$ until a muscle sample was collected [13, 36, 37].

\section{Library preparation, and mitochondrial genome sequencing using short reads}

Muscle was extracted from a pereiopod, and the tissue was immediately snap-frozen within a $50 \mathrm{ml}$ centrifuge tube located inside a $3 \mathrm{~L}$ plastic ice chest containing dry ice blocks $\left(-78.5^{\circ} \mathrm{C}\right)$. Within an hour of tissue extraction, the sample was transported to OMEGA Bioservices (Norcross, GA, USA). Total genomic DNA was purified from the muscle tissue using the OMEGA BIO-TEK ${ }^{\circ}$ E.Z.N.A. Blood and Tissue DNA Kit following the manufacturer's protocol. DNA concentration was measured using the QuantiFluor dsDNA system on a Quantus Fluorometer (Promega, Madison, WI, USA). A Kapa Biosystems HyperPrep kit (Kapa Biosystems, Wilmington, MA, USA) was used for whole genome library construction. Briefly, $1 \mu \mathrm{g}$ of genomic DNA was fragmented using a Bioruptor sonicator (Diagenode, Denville, NJ, USA). DNA fragment ends were repaired, 3' adenylated, and ligated to Illumina adapters. The resulting adapterligated libraries were PCR-amplified, Illumina indexes added, and pooled for multiplexed sequencing on an Illumina HiSeq X10 sequencer (Illumina, San Diego, CA, USA) using a pair-end 150 bp run format.

A total of 439,834,692 pairs of reads were generated and are available in the short-read archive (SRA) repository (accession number SRR13036344) at GeneBank.

\section{Mitochondrial genome assembly of Panulirus argus using short reads}

The mitochondrial genome of $P$. argus was assembled de novo using the NOVOPlasty pipeline v. 1.2.3 [47]. A single fragment of the cox1 gene available in GeneBank (MK308176) was used as a seed. A relatively large word (kmer) size of 39 was used during the assembly to profit from the large numbers of reads available. The newly assembled mitochondrial genome was then annotated in the MITOS and MITOS2 web servers [43] using the invertebrate genetic code. Annotation curation and start + stop codons corrections were performed using the Expasy translate tool (https://web.expasy.org/). Genome visualization was conducted with GCViewer (http://stothard. afns.ualberta.ca/cgview_server/ index.html - [48]). This assembly represents a trusted reference i.e., the 'ground truth' or 'golden standard' reference, that was used for benchmarking the accuracy of the de novo and reference-based assembled genomes using nanopore long reads alone.

\section{Library preparation, and mitochondrial genome sequencing using long reads}

Muscle tissue was extracted from a pereiopod and total genomic DNA (gDNA) was isolated using the QIAGEN ${ }^{\circ}$ DNeasy $^{\circ}$ Blood and Tissue Kit (QIAGEN ${ }^{\circ}$ USA) following the manufacturer's protocol. A total of five extractions were pooled together to obtain enough high molecular weight gDNA for preparing a single native barcoded ligation library using a modified version of the 'one-pot ligation protocol for Oxford Nanopore libraries' (https://protocols.io/ view/one-pot-ligation-protocol-foroxford-nanopore-libr-k9acz2e.html - [49]). In brief, gDNA concentration was measured using the Qubit 4 Fluorometer (Thermo Fisher Scientific) and purified for a first time using Ampure XP clean-up beads (Beckman Coulter, IN, USA) following the manufacturer's protocol. Next, a total of $200 \mathrm{fmol}$ purified gDNA was sheared (to $\sim 8000 \mathrm{bp}$ ) using a g-TUBE (Covaris, MA, USA) following the manufacturer's protocol. Sheared gDNA was purified for a second time using Ampure XP clean-up beads and then repaired using NEBNext FFPE DNA Repair Mix (New England Biolabs, MA, USA) and endprepared (poly-A adding) using NEBNext Ultra II End Repair/dA-Tailing Mix (New England Biolabs, MA, USA) following the manufacturer's protocol. Ligation of a native Oxford Nanopore barcode (NB10 from the EXP-NBD003 native barcoding kit, ONT, Oxford, UK) to the dA-tailed gDNA was conducted using NEBNext Ultra II Ligation Module (New England Biolabs, MA, USA) following the manufacturer's protocol. The resulting barcode-ligated library was pooled together with other libraries and BAM 1D sequencing adapters were ligated using NEBNext Ultra II Ligation Module (New England Biolabs, MA, USA) following the manufacturer's protocol. Lastly, the pooled libraries were purified for a last time using Ampure XP clean-up beads and multiplex-sequenced for $48 \mathrm{~h}$ on a MinION portable device with a MinION R9 flow cell (FLOMIN106, R9.4 chemistry) (ONT, Oxford, England) 


\section{Albacore [basecalling]}

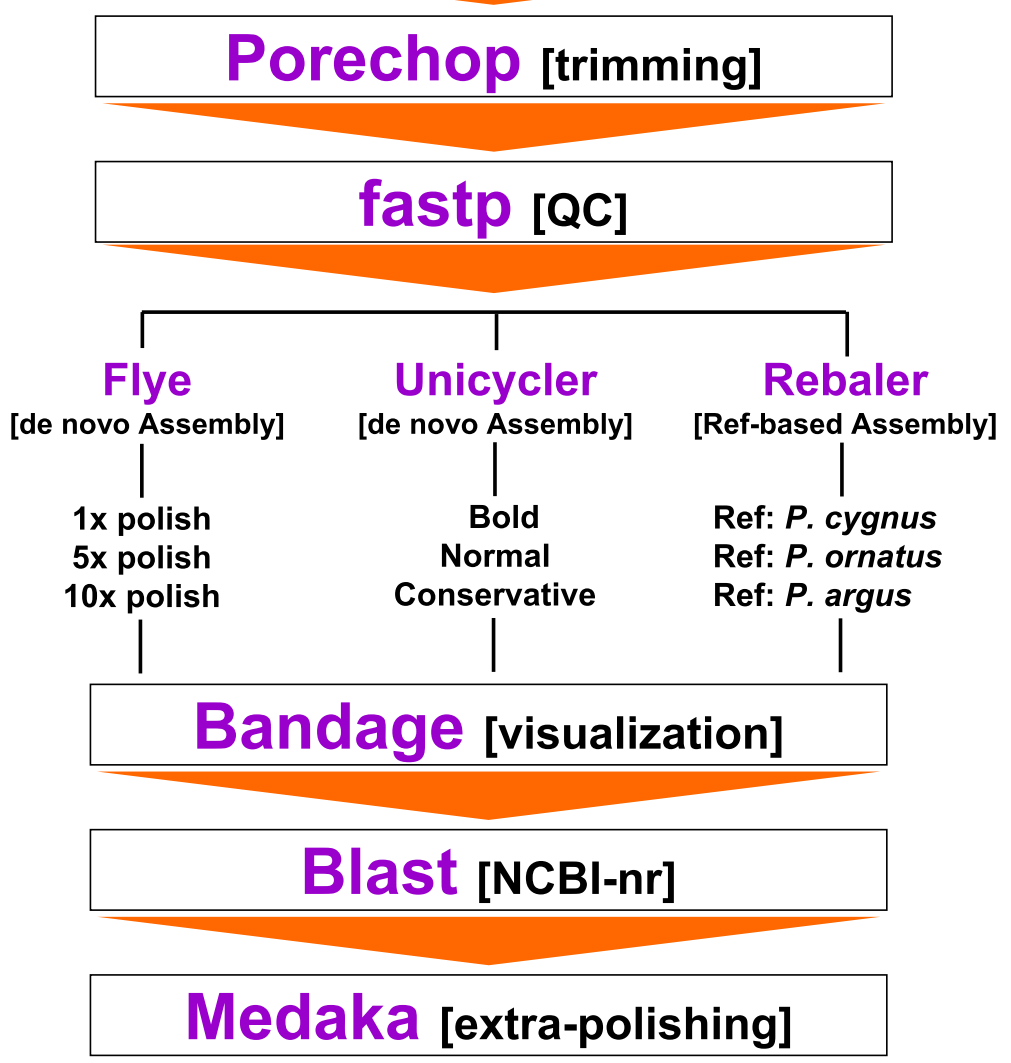

Fig. 6 Bioinformatics pipeline to assemble the mitochondrial genome of the Caribbean spiny lobster using nanopore long reads

controlled with the software MiniKnow. The raw signal (FAST5 files) was basecalled using the Albacore Sequencing Pipeline Software (ONT, Oxford, England) obtaining a total of 77,520 sequencing reads in FASTQ format (available in the short-read archive [SRA] repository at GenBank: accession number SRR13142981). The totality of these reads were used for assembling the mitochondrial genome of $P$. argus using different pipelines (Fig. 6).

De-novo assembly of Panulirus argus mitochondrial genome using long reads

Adapters were trimmed from read ends and reads with internal adapters were split into two using the software Porechop (https://github.com/rrwick/Porechop). The reads were then quality filtered with fastp [50] to retain only sequences with Q-score $\geq 6$, resulting in a total of 77,753 reads for assembly of the mitochondrial genome using three different de-novo pipelines; Canu 2.0.0 [39], Unicycler 0.4.8-1 [51], and Flye 2.8-0 [52] (Fig. 6).
Canu is a modification of the Celera Assembler [53] designed for high-noise single-molecule sequencing [39]. The hierarchical assembly pipeline in Canu comprises four steps: (i) detection of overlaps in the set of sequences, (ii) generation of a corrected sequence consensus, (iii) trimming corrected sequences, and (iv) assembly of trimmed corrected sequences [39]. Canu was executed twice, once with default options (but using genomeSize $=16 \mathrm{k}$ ) and a second time with modified parameters (genomeSize $=16 \mathrm{k}$, correctedError-Rate $=0.134$, corOutCoverage $=1000$, minOverlapLength $=300 \mathrm{bp}$ ) that was expected to optimize the retrieval of short circular molecule assemblies, including mitochondrial genomes ([39], see also [40]).

In the absence of short reads, the pipeline Unicycler [51] uses the software miniasm [54] that, in turn, relies on an overlap-and-layout algorithm, to concatenate sequences and assemble the longest possible set of unique contigs. Next, the software Racon [55] is used to 'polish' the final assembly. The number of polishing iterations is automatically selected by the program to increase the quality of the final assembly. Unicycler can be run in 
three modes: normal (the default), conservative, and bold. Conservative mode is least likely to produce a complete assembly but has a very low risk of misassembly. Bold mode is most likely to produce a complete assembly but carries greater risk of misassembly. Lastly, normal mode is intermediate regarding both completeness and misassembly risk (https://github.com/rrwick/ Unicycler). I ran each mode once to assemble the mitochondrial genome of $P$. argus. Importantly, Unicycler was chosen over the related program TryCycle (https:// github.com/rrwick/Trycycler) given the relatively low number of reads (and expected coverage $<25 \times$ ) available during this study.

Flye [52] first combines reads into error-prone 'disjointigs', then collapses repetitive sequences to make a repeat graph, and finally resolve the graph's repeats to produce a final set of contigs ([52], see also [42]). The last step involves polishing the final assembly using the program Flye polisher. By default, the Flye pipeline runs one polishing iteration. I ran Flye with 1, 5, and 10 iterations with the aim of improving the final assembly by correcting a small number of extra errors with increasing iterations.

I used the software Bandage [56] to visualize the assembly graph produced by each pipeline above. I predicted that a circularized sequence $\sim 15-16 \mathrm{kpb}$ in length would be observed among the contigs if any of the pipelines above successfully assembled and circularized the mitochondrial genome of $P$. argus. I also compared any other circularized and linear assembled contigs from each pipeline to the nucleotide nonredundant database in NCBI's GenBank and calculated the statistical significance of the matches to determine if any additional shorter incomplete mitochondrial reads were present among these contigs.

Other recently developed de novo assembly pipelines for long reads (i.e., Raven [https://github.com/idaholab/ raven], Minipolish [https://github.com/rrwick/Minipolish], and Shasta [https://github.com/chanzuckerberg/ shasta]) were not used here considering that these assemblers perform less efficiently than the ones used during this study (see [42] for details). Also, some of these assemblers, i.e., Shasta, are not available in Bioconda. Minipolish performs well compared to other pipelines but it uses a strategy very similar to that of Unicycler.

Lastly, I implemented a final 'extra polishing' step for each mitochondrial genome assembled with the pipelines above using the state-of-the-art program Medaka (https:// github. com/nanoporetech/medaka). Medaka creates a final consensus sequence $(=$ mitochondrial genome assembly) from nanopore sequencing reads using neural networks applied from a pileup of individual sequencing reads against a draft assembly. In this case, the draft assembly corresponds to the final assembly produced by the different aforementioned pipelines.

\section{Reference-based mitochondrial genome assembly of Panulirus argus using long reads}

The pipeline Rebaler (https://github.com/rrwick/Rebaler) was used for conducting reference-based assemblies of the mitochondrial genome of $P$. argus using the nanopore long reads. Rebaler first uses the program minimap2 [54] for aligning reads to a particular reference genome and then remove lower quality alignments (judged by total length, identity, and indel length) until the reference is minimally covered (any given position in the reference ends having a coverage of $1-2 x$, or zero if the reads failed to cover a section) (Fig. 6). Next, Rebaler replaces the reference sequence with the corresponding read fragments to produce an unpolished assembly and finally conducts multiple rounds of polishing with the software Racon using all reads to produce the best possible consensus sequence (https://github.com/rrwick/ Rebaler). I informed the pipeline that the reference genome was circular so that the contigs were 'rotated' (= change in the starting position) between polishing rounds to ensure that all parts of the mitochondrial genome, including the ends, were well polished (https:// github.com/rrwick/Rebaler).

I executed Rebaler first using the reference genome of $P$. argus generated during this study and then again twice with two other reference mitochondrial genomes available in Genbank; P. cygnus (NC_028024.1) and P. ornatus (NC_014854.1). Panulirus ornatus is more distantly related to P. argus than P. cygnus according to phylogenetic studies based on two fragments of the mitochondrial genome [44]. Thus, the use of three different reference genomes permitted exploring any effect of the reference genome on the accuracy of the final assembly by Rebaler. Previous studies have shown the absence of differences in gene synteny among mitochondrial genomes in the genus Panulirus ([11] and references therein).

As with the de novo assembled genomes, I also implemented a final 'polishing' step for each mitochondrial genome assembled with Rebaler (if any) using the program Medaka (https://github. com/nanoporetech/medaka).

\section{Accuracy of long-read mitochondrial genome assemblies}

To assess the accuracy of each mitochondrial genome assembled with the different pipelines with and without a final polishing with the program Medaka, I used four metrics: number of contigs, number of bases (length) in the assembly, coverage, and identity. Patristic (p-) distance is herein implemented as a measure of read identity with high values indicating low read accuracy and low $p$-values indicating high read accuracy. A p-value of zero indicates identical short-read reference and longread assembled mitogenomes. Patristic p-distance between the reference genome and each of the genomes assembled with long reads was calculated after aligning 
each of the long-read assembled mitochondrial genomes to the reference genome with the program Muscle [57] as implemented in the software MEGAX [58].

I calculated additional proxies for accuracy and quantified long-read assembly error in a manner similar to that of [59]. After each long-read assembly (with and without 'extra polishing' using Medaka) was aligned to the reference assembly, errors were classified as single, double, triple, quadruple, or quintuple "homopolymer insertions' or 'homopolymer deletions' if the error added or removed a single, two, three, four or five bases from a homopolymer (i.e. multiple consecutive appearances of the same nucleotide) three or more bases in length. Other errors that did not fit with any of the aforementioned classification categories were classified as 'substitution', 'single insertion', 'short insertion ( $<3 \mathrm{bp})$ ', 'single deletion', and 'short deletion $(<3 \mathrm{pb})$ '.

\section{Annotation of long-read mitochondrial assemblies}

For each de novo and reference-based pipeline that successfully assembled the mitochondrial genome of $P$. argus, irrespective of accuracy, genome annotation was conducted using MITOS and MITOS 2 [43]. The presence/absence and number (if any) of stop codons and interruptions in the open reading frame of the PCGs was recorded as it also represents an additional proxy for accuracy explored herein.

\section{Phylogenomic and barcoding utility of long-read mitochondrial assemblies}

I explored if the newly assembled genomes of $P$. argus using long reads alone were useful for studies focusing on phylogenomics and barcoding. I predicted that, in both mitophylogenomic and barcoding analyses, the long-read assembled genomes will cluster with the reference short-read assembly genome and will segregate from other closely and distantly related mitochondrial genome sequences from species in the same genus, family, and superorder available in Genbank.

To accomplish this goal, the totality of the mitochondrial genomes $(N=18)$ belonging to the order Achelata, to which $P$. argus belongs, were retrieved from GenBank (available as of 0824 2020). Each of the PCGs from the species above plus those from the different mitochondrial genomes generated using long reads and short reads (reference genome) in this study were aligned with default parameters in the program Muscle [57] as implemented in the software MEGA X [58]. The final alignment, comprising $11,187 \mathrm{bp}$, was submitted to the IQTREE 1.6.12 web server (http://iqtree.cibiv.univie.ac.at/) for Maximum Likelihood (ML) analysis [60]. During the analyses, one species of caridean shrimp (order Caridea, Synalpheus microneptunus), one species of stenopodid shrimp (order Stenopodidae, Stenopus hispidus), and two species of clawed lobsters (order Polychelidae, Polycheles baccatus and P. typhlops) were used as outgroups. Selection of a base substitution model that best fits each dataset was conducted with ModelFinder [61] as implemented in IQ-TREE. The optimal models found by ModelFinder (selected with the Bayesian Information Criterion) were the $\mathrm{K} 3 \mathrm{Pu}+\mathrm{F}+\mathrm{I}+\mathrm{G} 4, \mathrm{HKY}+\mathrm{F}+\mathrm{I}+\mathrm{G} 4$, $\mathrm{GTR}+\mathrm{F}+\mathrm{I}+\mathrm{G} 4, \quad \mathrm{TIM} 2+\mathrm{F}+\mathrm{I}+\mathrm{G} 4, \quad \mathrm{TIM}+\mathrm{F}+\mathrm{I}+\mathrm{G} 4$, $\mathrm{TIM} 2+\mathrm{F}+\mathrm{I}+\mathrm{G} 4, \quad \mathrm{TVM}+\mathrm{F}+\mathrm{I}+\mathrm{G} 4, \quad \mathrm{TPM} 2 \mathrm{u}+\mathrm{F}+\mathrm{I}+$ G4, HKY + F + I + G4, TVM + F + I + G4, HKY + F + G4, $\mathrm{GTR}+\mathrm{F}+\mathrm{I}+\mathrm{G} 4$, and $\mathrm{K} 3 \mathrm{Pu}+\mathrm{F}+\mathrm{G} 4$ for atp6, atp 8 , cox1, cox2, cox3, cytb, nad1, na2; nad3, nad4, nad4l, $n a d 5$, and nad6, respectively. All the parameters used for the ML analyses were those of the default options in IQ-TREE and 1000 bootstrap replications were conducted to estimate support for each node in the Maximum Likelihood tree [60].

Lastly, the barcoding utility of the cox 1 gene assembled with long reads (both de novo and reference-based) was tested. Even if not fully accurate (see results), the assembled gene might permit the reliable identification of the sequenced specimen as $P$. argus and it might permit reliably differentiating the specimen from other representatives in the same genus and family within the Decapoda infraorder Achelata (clawed lobsters). Alternatively, the errors might constrain the proper identification of the specimen as $P$. argus. If the assembled gene is useful for barcoding studies, then I predicted that the cox 1 gene fragments produced by the de novo and reference-based assemblies will cluster together with the short-read assembled $\operatorname{cox} 1$ gene fragment as well as with other $\operatorname{cox} 1$ fragments from the same species available in GenBank and the latter fragments will segregate from others belonging to closely related congeneric and confamiliar species and even from more distantly related species in the same order Achelata.

To accomplish this goal, a total of 1972 cox1 sequences were retrieved from GenBank (available as of 08 24 2020). From this initial set of sequences, I eliminated 49 due to the presence of ambiguous ' $N$ ' nucleotides along the fragment that likely imply poor sequencing quality. Next, the totality of the remaining sequences plus the $\operatorname{cox} 1$ gene fragment generated using long reads and short reads (reference genome) were aligned with default parameters in the program Clustal Omega [59, 62] as implemented in the web server EMBO (https:// www.ebi.ac.uk/Tools/ msa/clustalo/). The final alignment consisted of $1539 \mathrm{bp}$, had no indels (other than those produced by the cox 1 gene assembled with long reads) and was unambiguous. Considering that different barcoding studies use different adapters to sequence different portions of the $\operatorname{cox} 1$ gene, I divided the final alignment into three portions (positions 1-500, 501-1000, and 1001-1539 bp) and eliminated all sequences shorter 
than $250 \mathrm{bp}$ from each of the new datasets. The aforementioned procedure resulted in a total of 1899, 185, and 210 terminals in the first, second, and third dataset, respectively.

Molecular phylogenetic analyses were conducted separately for each dataset. During the analyses, species of lobsters not belonging to the superfamily Palinuroidea within the infraorder Achelata were used as outgroups. Each aligned dataset was submitted to the IQ-TREE 1.6.12 web server (http://iqtree.cibiv.univie.ac.at/) for Maximum Likelihood (ML) analysis [60]. Selection of a base substitution model that best fits each dataset was conducted with ModelFinder [61] as implemented in IQ-TREE. The optimal models found by ModelFinder (selected with the Bayesian Information Criterion) were the $\mathrm{TIM}+\mathrm{F}+\mathrm{I}+\mathrm{G} 4, \mathrm{HKY}+\mathrm{F}+\mathrm{I}+\mathrm{G} 4$, and TPM2 + F + $\mathrm{I}+\mathrm{G} 4$ for the first, second, and third dataset, respectively. All the parameters used for the ML analyses were those of the default options in IQ-TREE and 1000 bootstrap replications were conducted to estimate support for each node in each Maximum Likelihood tree [60].

\section{Acknowledgments}

The author would like to thank Tom Matthews (FFWCC) and his team at the Florida Fish and Wildlife Conservation Commission for their help in collecting specimens. Many thanks to Dr. Vince P. Richards for bioinformatics support during the development of this project. Many thanks to Tim Cronin for improving the English language of this manuscript. Many thanks to Dr. Carlos Saavedra and one anonymous referee whose comments permitted to improve earlier versions of this manuscript. This study would have not been possible without all the help and knowledge provided by Josh Quick, Nick Loman, and Matt Loose during the course" PoreCamp - Vancouver" at the Michael Smith Laboratories, University of British Columbia, Canada.

\section{Author's contributions}

J. Antonio Baeza: Conceptualization, Methodology, Analysis, Data curation, Writing, Original draft preparation. Visualization, Writing, Reviewing, and Editing. The author read and approved the final manuscript.

\section{Funding}

This research did not receive any specific grant from funding agencies in the public, commercial, or not-for-profit sectors.

\section{Availability of data and materials}

All datasets on which the conclusions of the manuscript rely are presented in the main paper.

Raw reads were submitted to the SRA database of NCBI with accession numbers SRR13036344 and SRR13142981.

\section{Ethics approval and consent to participate}

No approval by an ethical committee was required to accomplish the goals of the present study because experimental work was conducted with an unregulated marine invertebrate. Florida Fish and Wildlife Conservation Commission gently granted permission for sampling lobster specimens from the wild.

\section{Consent for publication}

not applicable.

\section{Competing interests}

The author declares no competing interests.

\section{Author details}

'Department of Biological Sciences, Clemson University, 132 Long Hall, Clemson, SC 29634, USA. ${ }^{2}$ Smithsonian Marine Station at Fort Pierce, 701
Seaway Drive, Fort Pierce, Florida 34949, USA. ${ }^{3}$ Departamento de Biología Marina, Facultad de Ciencias del Mar, Universidad Católica del Norte, Larrondo 1281, Coquimbo, Chile.

Received: 5 October 2020 Accepted: 28 November 2020

Published online: 09 December 2020

\section{References}

1. Taanman JW. The mitochondrial genome: structure, transcription, translation and replication. Biochimica et Biophysica Acta (BBA)-Bioenergetics. 1999; 1410:103-23.

2. McBride HM, Neuspiel M, Wasiak S. Mitochondria: more than just a powerhouse. Curr Biol. 2006;16:R551-60.

3. Bernt M, Bleidorn C, Braband A, Dambach J, Donath A, Fritzsch G, Golombek A, Hadrys $H$, Jühling F, Meusemann K, Middendorf M. A comprehensive analysis of bilaterian mitochondrial genomes and phylogeny. Mol Phylogen Evol. 2013;69:352-64.

4. John U, Lu Y, Wohlrab S, Groth M, Janouškovec J, Kohli GS, Mark FC, Bickmeyer U, Farhat S, Felder M, Frickenhaus S. An aerobic eukaryotic parasite with functional mitochondria that likely lacks a mitochondrial genome. Science Adv. 2019;5(4):eaav1110

5. Doucet-Beaupré $H$, Breton S, Chapman EG, Blier PU, Bogan AE, Stewart DT, Hoeh WR. Mitochondrial phylogenomics of the Bivalvia (Mollusca): searching for the origin and mitogenomic correlates of doubly uniparental inheritance of mtDNA. BMC Evol Biol. 2010;10(1):50.

6. Ladoukakis ED, Zouros E. Evolution and inheritance of animal mitochondrial DNA: rules and exceptions. J Biol Res - Thessaloniki. 2017;24:1-7.

7. Ballard JWO, Rand DM. The population biology of mitochondrial DNA and its phylogenetic implications. Annu Rev Ecol Evol Syst. 2005;36:621-42.

8. Galtier N, Nabholz B, Glémin S, Hurst GDD. Mitochondrial DNA as a marker of molecular diversity: a reappraisal. Mol Ecol. 2009;18:4541-50.

9. Avise JC. Phylogeography: the history and formation of species. Cambridge, MA: Harvard University Press; 2000.

10. Lou RN, Fletcher NK, Wilder AP, Conover DO, Therkildsen NO, Searle JB. Full mitochondrial genome sequences reveal new insights about postglacial expansion and regional phylogeographic structure in the Atlantic silverside (Menidia menidia). Mar Biol. 2018;165(8):124.

11. Baeza JA, Sepulveda FA, Gonzalez MT. The complete mitochondrial genome and description of a new cryptic species of Benedenia Diesing, 1858 (Monogenea: Capsalidae), a major pathogen infecting the yellowtail kingfish Seriola lalandi Valenciennes in the south-East Pacific. Parasites Vectors. 2019; 12:490.

12. Veldsman WP, Wang Y, Niu J, Baeza JA, Chu KH. Characterization of the complete mitochondrial genome of a coconut crab, Birgus latro (Linnaeus, 1767)(Decapoda: Anomura: Coenobitidae), from Okinawa. Japan J Crustac Biol. 2020:40:390-400.

13. Baeza JA. The complete mitochondrial genome of the Caribbean spiny lobster Panulirus argus. Sci Rep. 2018:8(1):17690.

14. Chak STC, Barden P, Baeza JA. The complete mitochondrial genome of the eusocial sponge-dwelling snapping shrimp Synalpheus microneptunus. Sci Rep. 2020;10:7744.

15. Ma X, Agudelo P, Richards VP, Baeza JA. The complete mitochondrial genome of the Columbia lance nematode, Hoplolaimus columbus, a major agricultural pathogen in North America. Parasites Vectors. 2020;13:321

16. Anderson S, Bankier AT, Barrell BG, de Bruijn MH, Coulson AR, Drouin J, Eperon IC, Nierlich DP, Roe BA, Sanger F, Schreier PH. Sequence and organization of the human mitochondrial genome. Nature. 1981;290:457-65.

17. Faria NR, Kraemer MU, Hill SC, De Jesus JG, Aguiar RS, lani FC, Xavier J, Quick J, Du Plessis L, Dellicour S, Theze J. Genomic and epidemiological monitoring of yellow fever virus transmission potential. Science. 2018;361:894-9.

18. Baeza JA, Behringer DC. Integrative taxonomy of the ornamental 'peppermint' shrimp public market and population genetics of Lysmata boggessi, the most heavily traded species worldwide. PeerJ. 2017;5:e3786.

19. Jain M, Koren S, Miga KH, Quick J, Rand AC, Sasani TA, Tyson JR, Beggs AD, Dilthey AT, Fiddes IT, Malla S. Nanopore sequencing and assembly of a human genome with ultra-long-read. Nature Biotech. 2018;36:338-45.

20. Rhoads A, Au KF. PacBio sequencing and its applications. Genomics Proteomics Bioinformatics. 2015;13:278-89.

21. Rang FJ, Kloosterman WP, de Ridder J. From squiggle to basepair: computational approaches for improving nanopore sequencing read accuracy. Genome Biol. 2018;19(1):90. 
22. Goldstein S, Beka L, Graf J, Klassen JL. Evaluation of strategies for the assembly of diverse bacterial genomes using MinION long-read sequencing. BMC Genomics. 2019;20(1):23.

23. Wang WW, Schalamun M, Morales-Suarez A, Kainer D, Schwessinger B, Lanfear R. Assembly of chloroplast genomes with long- and short-read data: a comparison of approaches using Eucalyptus pauciflora as a test case. BMC Genomics. 2018;19:977.

24. Gan HM, Grandjean F, Jenkins TL, Austin CM. Absence of evidence is not evidence of absence: Nanopore sequencing and complete assembly of the European lobster (Homarus gammarus) mitogenome uncovers the missing nad2 and a new major gene cluster duplication. BMC Genomics. 2019;20(1):1-11.

25. Gan HM, Linton SM, Austin CM. Two reads to rule them all: Nanopore long read-guided assembly of the iconic Christmas Island red crab, Gecarcoidea natalis (Pocock, 1888), mitochondrial genome and the challenges of AT-rich mitogenomes. Mar Genomics. 2019;45:64-71.

26. Gao K, Li J, Khan WU, Zhao T, Yang X, Yang X, Guo B, An X. Comparative genomic and phylogenetic analyses of Populus section Leuce using complete chloroplast genome sequences. Tree Genet Genomes. 2019;15(3):32.

27. Kang HI, Lee HO, Lee IH, Kim IS, Lee SW, Yang TJ, Shim D. Complete chloroplast genome of Pinus densiflora Siebold \& Zucc. and comparative analysis with five pine trees. Forests. 2019;10(7):600

28. Belser C, Istace B, Denis E, Dubarry M, Baurens FC, Falentin C, Genete M, Berrabah W, Chèvre AM, Delourme R, Deniot G. Chromosome-scale assemblies of plant genomes using nanopore long reads and optical maps. Nature Plants. 2018:4:879-87.

29. Koren S, Schatz MC, Walenz BP, Martin J, Howard JT, Ganapathy G, Wang Z, Rasko DA, McCombie WR, Jarvis ED, Phillippy AM. Hybrid error correction and de novo assembly of single-molecule sequencing reads. Nature Biotech. 2012;30:693-700.

30. Leggett RM, Clark MD. A world of opportunities with nanopore sequencing. J Exp Bot. 2017:68:5419-29.

31. Jansen HJ, Liem M, Jong-Raadsen SA, Dufour S, Weltzien FA, Swinkels W, Koelewijn A, Palstra AP, Pelster B, Spaink HP, Van den Thillart GE. Rapid de novo assembly of the European eel genome from nanopore sequencing reads. Sci Rep. 2017;7(1):1-3.

32. Franco-Sierra ND, Díaz-Nieto JF. Rapid mitochondrial genome sequencing based on Oxford Nanopore sequencing and a proxy for vertebrate species identification. Ecol Evol. 2020;10:3544-60.

33. Higgs ND, Newton J, Attrill MJ. Caribbean spiny lobster fishery is underpinned by trophic subsidies from chemosynthetic primary production. Curr Biol. 2016;26:3393-8.

34. Holthuis LB. FAO species catalogue. Marine lobsters of the world. An annotated and illustrated catalogue of species of interest to fisheries known to date in FAO fisheries Synopsis. FAO. BMC Zoology. 1991;13.

35. Kozma MT, Schmidt M, Ngo-Vu H, Sparks SD, Senatore A, Derby CD. Chemoreceptor proteins in the Caribbean spiny lobster, Panulirus argus: expression of ionotropic receptors, gustatory receptors, and TRP channels in two chemosensory organs and brain. PLoS One. 2018;13(9):e0203935.

36. Baeza JA, Umaña-Castro R, Mejia-Ortiz LM. Historical demography of the Caribbean spiny lobster Panulirus argus (Latreille, 1804)(Decapoda: Achelata: Palinuridae) in the Florida keys, USA inferred using single nucleotide polymorphisms (SNPs). J Crustac Biol. 2019;39:378-85.

37. Baeza JA, MacManes M. De novo assembly and functional annotation of the heart+ hemolymph transcriptome in the Caribbean spiny lobster Panulirus argus. Mar Genomics. 2020:54:100783.

38. Subramaniam K, Behringer DC, Bojko J, Yutin N, Clark AS, Bateman KS, van Aerle R, Bass D, Kerr RC, Koonin EV, Stentiford GD. A new family of DNA viruses causing disease in crustaceans from diverse aquatic biomes. Mbio. 2020;11(1).

39. Koren S, Walenz BP, Berlin K, Miller JR, Bergman NH, Phillippy AM. Canu: scalable and accurate long-read assembly via adaptive k-mer weighting and repeat separation. Genome Res. 2017;27:722-36.

40. Scheunert A, Dorfner M, Lingl T, Oberprieler C. Can we use it? On the utility of de novo and reference-based assembly of Nanopore data for plant plastome sequencing. PLoS One. 2020;15(3):e0226234.

41. Laehnemann D, Borkhardt A, McHardy AC. Denoising DNA deep sequencing data-high-throughput sequencing errors and their correction. Brief Bioinformatics. 2016:17:154-79.

42. Wick RR, Holt KE. Benchmarking of long-read assemblers for prokaryote whole genome sequencing. F1000Research. 2019;8.
43. Bernt M, Donath A, Jühling F, Externbrink F, Florentz C, Fritzsch G, Pütz J, Middendorf M, Stadler PF. MITOS: improved de novo metazoan mitochondrial genome annotation. Mol Phylogen Evol. 2013;69:313-9.

44. Ptacek MB, Sarver SK, Childress MJ, Herrnkind WF. Molecular phylogeny of the spiny lobster genus Panulirus (Decapoda: Palinuridae). Mar Freshw Res. 2001;52:1037-47.

45. Truelove NK, Kough AS, Behringer DC, Paris CB, Box SJ, Preziosi RF, Butler $\mathrm{MJ}$. Biophysical connectivity explains population genetic structure in a highly dispersive marine species. Coral Reefs. 2017;36:233-44.

46. Naro-Maciel E, Reid B, Holmes KE, Brumbaugh DR, Martin M, DeSalle R. Mitochondrial DNA sequence variation in spiny lobsters: population expansion, panmixia, and divergence. Mar Biol. 2011;158:2027-41.

47. Dierckxsens N, Mardulyn P, Smits G. NOVOPlasty: de novo assembly of organelle genomes from whole genome data. Nucleic Acids Res. 2016;45:e18.

48. Grant JR, Stothard P. The CGView server: a comparative genomics tool for circular genomes. Nucleic Acids Res. 2008;36(suppl. 2):W181-4.

49. Quick, J. 2020. https://protocols.io/ view/one-pot-ligation-protocol-foroxford-nanopore-libr-k9acz2e.html.

50. Chen S, Zhou Y, Chen Y, Gu J. Fastp: an ultra-fast all-in-one FASTQ preprocessor. Bioinformatics. 2018;34:i884-90.

51. Wick RR, Judd LM, Gorrie CL, Holt KE. Unicycler: resolving bacterial genome assemblies from short and long sequencing reads. PLoS Comp Biol. 2017:13: e1005595.

52. Kolmogorov M, Yuan J, Lin Y, Pevzner PA. Assembly of long, error-prone reads using repeat graphs. Nature Biotech. 2019;37:540-6.

53. Myers EW, Sutton GG, Delcher AL, Dew IM, Fasulo DP, Flanigan MJ, Kravitz SA, Mobarry CM, Reinert KH, Remington KA, Anson EL. A whole-genome assembly of Drosophila. Science. 2000;287:2196-204.

54. Li H. Minimap and miniasm: fast mapping and de novo assembly for noisy long sequences. Bioinformatics. 2016;32:2103-10.

55. Vaser R, Sović I, Nagarajan N, Šikić M. Fast and accurate de novo genome assembly from long uncorrected reads. Genome Res. 2017;27:737-46.

56. Wick RR, Schultz MB, Zobel J, Holt KE. Bandage: interactive visualization of de novo genome assemblies. Bioinformatics. 2015;31:3350-2.

57. Edgar RC. MUSCLE: multiple sequence alignment with high accuracy and high throughput. Nucleic Acids Res. 2004;32:1792-7.

58. Kumar S, Stecher G, Li M, Knyaz C, Tamura K. MEGA X: molecular evolutionary genetics analysis across computing platforms. Mol Biol Evol. 2018;35:1547-9.

59. Wick RR, Judd LM, Holt KE. Performance of neural network basecalling tools for Oxford Nanopore sequencing. Genome Biol. 2019;20(1):129.

60. Trifinopoulos J, Nguyen LT, von Haeseler A, Minh BQ. W-IQ-TREE: a fast online phylogenetic tool for maximum likelihood analysis. Nucleic Acids Res. 2016:44(W1):W232-5.

61. Kalyaanamoorthy S, Minh BQ, Wong TK, von Haeseler A, Jermiin LS. ModelFinder: fast model selection for accurate phylogenetic estimates. Nat Methods. 2017;14:587-9.

62. Siervers F, Wilm A, Dineen D, Gibson TJ, Karplus K, Li W, Lopez R, McWilliam H, Remmert M, Söding J, Thompson JD. Fast, scalable generation of highquality protein multiple sequence alignments using Clustal Omega. Mol Syst Biol. 2011;7(1):539.

\section{Publisher's Note}

Springer Nature remains neutral with regard to jurisdictional claims in published maps and institutional affiliations.

Ready to submit your research? Choose BMC and benefit from:

- fast, convenient online submission

- thorough peer review by experienced researchers in your field

- rapid publication on acceptance

- support for research data, including large and complex data types

- gold Open Access which fosters wider collaboration and increased citations

- maximum visibility for your research: over $100 \mathrm{M}$ website views per year

At $\mathrm{BMC}$, research is always in progress.

Learn more biomedcentral.com/submissions 